\title{
Isolated laryngeal tuberculosis in a 10-year-old boy: A case report
}

\author{
Santosh Kumar Swain', Alok Das', Mahesh Chandra Sahu² \\ 'Department of Otorhinolaryngology, IMS and SUM Hospital, Siksha "O" Anusandhan University, K8, Kalinganagar, \\ Bhubaneswar, Odisha, India \\ 2Directorate of Medical Research, IMS and SUM Hospital, Siksha "O" Anusandhan University, K8, Kalinganagar, Bhubaneswar, \\ Odisha, India
}

\section{ABSTRACT}

Tuberculosis is a major health problem in developing and underdeveloped countries of the world. Tuberculosis is a chronic granulomatous disease caused by Mycobacterium tuberculosis, and its incidence is rising due to increasing human immunodeficiency virus (HIV) infections. It usually affects lungs, but isolated laryngeal tuberculosis in a paediatric patient is a rare incidence. We are presenting a case of isolated laryngeal tuberculosis in a 10-year-old boy with clinical presentation of hoarseness of voice and dry irritating cough. The patient was treated with antitubercular treatment and recovery was successful with complete regression of the disease.

\section{KEY WORDS:}

isolated, larynx, tuberculosis, antitubercular therapy, paediatric patient.

\section{INTRODUCTION}

Tuberculosis is a major public health problem in developing and underdeveloped countries. Tuberculosis is a granulomatous infection caused by the Mycobacterium tuberculosis. It often affects the lungs, and pulmonary tuberculosis accounts for $80 \%$ of cases [1]; however, it can involve any other organ of the human body. Laryngeal tuberculosis is a rare type of extrapulmonary tuberculosis, which constitutes less than $1 \%$ of all types of tuberculosis cases [2]. Low immunity of the body or the increase in multidrug-resistant tuberculosis may be the cause of laryngeal tuberculosis. Approximately 530,000 children are suffering with tuberculosis in developing countries around the world [3]. The extrapulmonary involvement of the tuberculosis ranges from 30 to $40 \%$ [4].

The larynx is an uncommon site for tuberculosis among children. Laryngeal tuberculosis may manifest from mild hoarseness of voice to severe odynophagia and dyspnoea due to laryngeal oedema and granulations. Paediatricians or otorhinolaryngologists should be familiar with the unusual site of the larynx for tuberculosis as worldwide recrudescence of tuberculosis because of the spread of human immunodeficiency virus (HIV) infection and the fact that it can be confused with laryngeal cancer. We present a case of isolated laryngeal tuberculosis in a 10-year-old boy presenting with hoarseness of voice and dry cough.

\section{CASE REPORT}

A 10-year-old boy attended an outpatient department of otorhinolaryngology for hoarseness of voice and irritating dry cough for the preceding three months. He had been treated by a local paediatrician with conservative treatment along with a course of oral steroids, following which the symptoms become worse. He had no history of upper respiratory tract infections, fever, or weight loss.

\section{ADDRESS FOR CORRESPONDENCE:}

Santosh Kumar Swain, Department of Otorhinolaryngology, IMS and SUM Hospital, Bhubaneswar, Odisha,

India, e-mail: santoshvoltaire@yahoo.co.in 
There was no history of HIV infection in the child and he showed a negative result in serological test. He was vaccinated (BCG) against tuberculosis. Chest X-ray was normal. Fibreoptic nasopharyngolaryngoscopy was performed, which showed irregular and congested lesions at the anterior part of the glottis, which was more marked at the anterior end of the left vocal cord (Fig. 1). A computed tomography (CT) scan showed mild thickening of the left vocal cord. The biopsy was planned and done under general anaesthesia and histopathological examination revealed granulomatous lesions with caseations (Fig. 2). Sputum test for acid fast bacilli (AFB) was positive. For confirmation we sent for a culture in Löwenstein-Jensen (LJ) medium, and it revealed a positive result within two weeks. Routine blood investigations were within normal limits except for a slightly increased erythrocyte sedimentation rate. The patient was given antitubercular therapy (ATT) consisting of four drugs: isoniazid, rifampicin, pyrazinamide, and ethambutol, for two months followed by isoniazid and rifampicin for four months. After two months of therapy, symptomatically the patient improved. After completion of therapy the patient was examined and showed normal larynx.

\section{DISCUSSION}

Primary laryngeal tuberculosis in a paediatric patient is an extremely rare clinical entity and is often caused by direct invasion of inhaled Mycobacterium tuberculosis bacilli [5]. Secondary involvement of the larynx occurs by cough and expectoration from the tracheobronchial tree or via the blood stream from somewhere other than the lungs [6]. Lymphatic spread to the larynx is rare. The

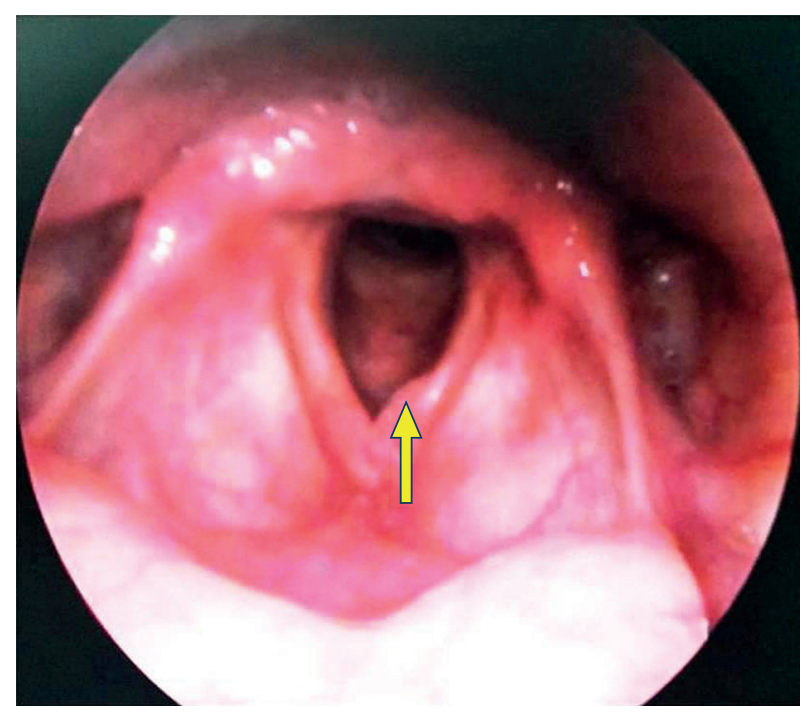

FIGURE 1. Endoscopic picture of larynx showing tubercular lesion at anterior end of left vocal cord (arrow mark)

prevalence of laryngeal sites affected by tuberculosis are in decreasing order as follows: true vocal folds (50-70\%), false cords (40-50\%), and epiglottis, aryepiglottic folds, arytenoids, posterior commissure, and/or subglottis (1015\%) [6]. The tubercular lesions in the larynx may appear as ulcerative, ulcerofungative, polypoidal, or nonspecific inflammatory [7]. Congestion of a unilateral vocal cord should alert for possibility of a tubercular lesion. Laryngeal tuberculosis often presents with hoarseness of voice (80-100\%) and odynophagia (50-67\%). It may lead to severe dyspnoea and stridor due to laryngeal oedema and granulations. It can affect any part of the larynx [8].

The vocal cords of the larynx are commonly affected in laryngeal tuberculosis. The laryngeal tuberculosis
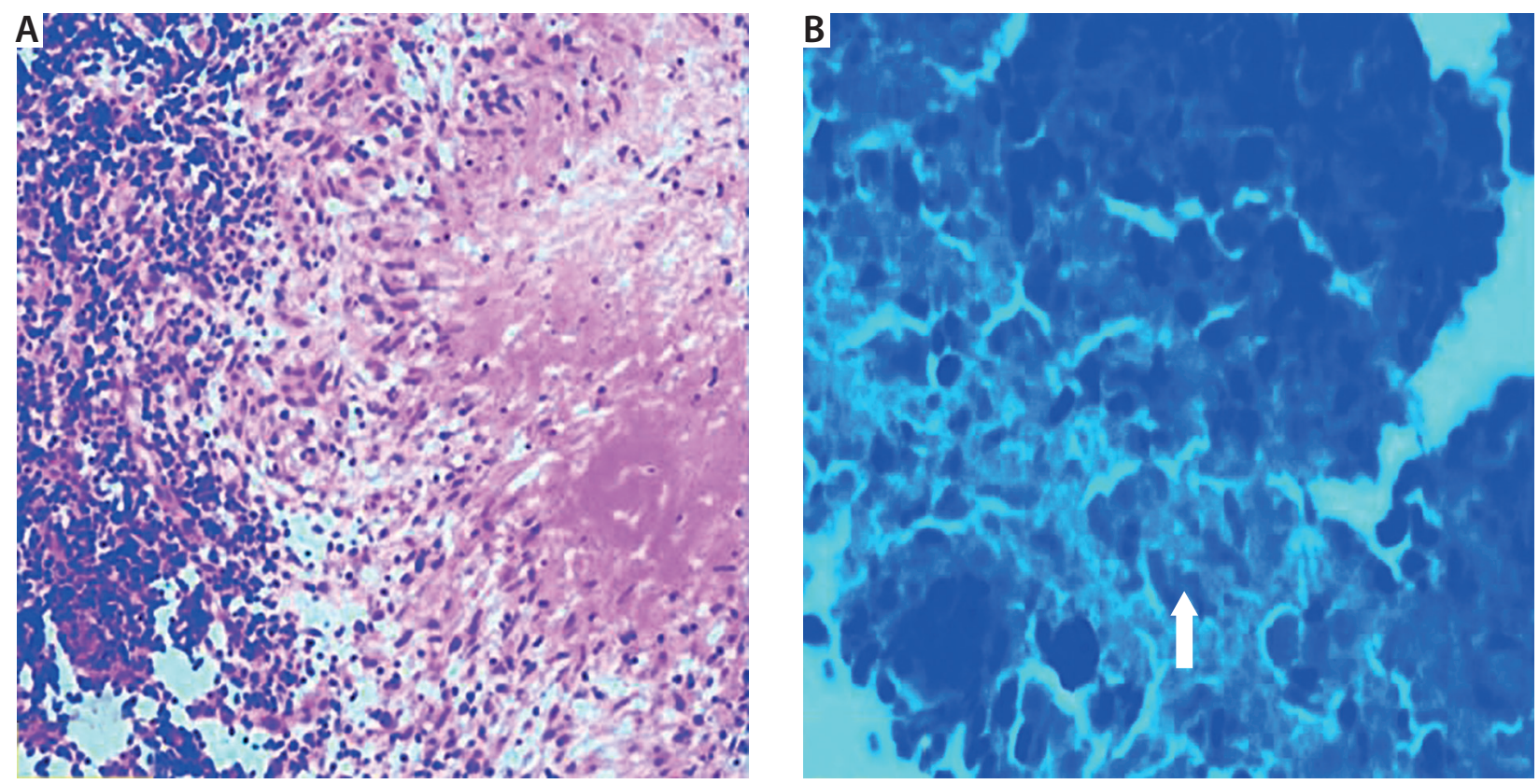

FIGURE 2. A - Histopathological examination showing granuloma formation with caseous necrosis. B - Z\&N (Ziehl-Nielsen) staining from biopsy specimen showing acid-fast bacilli (arrow mark) 
is characterised by oedema, hyperaemia, or ulcerative lesions in the larynx. Laryngeal tuberculosis has no gender or age predilection, but in paediatric age, laryngeal tuberculosis may not reveal abnormalities in chest X-ray [5]. In our case, chest X-ray of the patient was normal, which may mislead the clinician and delay the diagnosis. Fibreoptic nasopharyngolaryngoscopy shows mucosal thickening, ulcerations, or polypoidal mass. Malignancies such as squamous cell carcinoma and tuberculosis may coexist in the same patient, so biopsy is essential in such patients [6]. Pulmonary tuberculosis with scar tissue in the lungs may cause traction neuropathy by affecting the recurrent laryngeal nerve, leading to vocal cord palsy [9]. Differentiation between laryngeal carcinoma and laryngeal tuberculosis is often difficult. Previously, laryngeal tuberculosis was due to complications of pulmonary tuberculosis. Tuberculosis of the larynx is often confused with syphilis, fungal laryngitis, and granulomatous lesions like Wegner's granulomatosis and sarcoidosis. In clinical practice, the most important differential diagnosis of laryngeal tuberculosis is laryngeal carcinoma. Both laryngeal tuberculosis and laryngeal carcinoma have similar clinical, endoscopic, and radiological features [10].

The diagnosis of laryngeal tuberculosis is based on pathological and microbiological findings of a biopsy specimen collected from the larynx. Diagnosis of laryngeal tuberculosis is confirmed by histopathological examinations with chronic granulomatous inflammatory exudates with or without caseating necrosis. In some cases when the histopathological picture shows an absence of caseations, the diagnosis is confirmed by recovery of the patients under anti-tubercular therapy. Routine blood investigations like total white cell counts, differential count, and ESR are done in all cases. A tuberculin test is also advised in all cases. Sputum microscopy for AFB is positive in around $20 \%$ of cases of laryngeal tuberculosis, and most of the chest X-ray findings are persistent with pulmonary tuberculosis [11]. Ziehl-Neelsen staining may directly show acid-fast bacilli. Microbiological culture and drug sensitivities of the infective strain are not usually done in practice, whereas multiple repeated biopsies are often used to confirm the diagnosis and start the treatment [12]. ZN staining and LJ medium growth are often used for diagnosis, whereas LJ medium growth is considered the gold standard, but sometimes it gives false negative results due to the low number of bacilli. Samples that are not positive $\mathrm{ZN}$ stain and LJ medium growth are usually sent for molecular diagnosis of polymerase chain reaction (PCR). The PCR test is often used in developed countries for diagnosis of tuberculosis. The detection of Mycobacterium tuberculosis is increased from $2 \%$ to $17 \%$ on culture to $89-100 \%$ in PCR [13]. In cases of strong clinician suspicion of tuberculosis with negative cultures, samples can be sent for PCR test [14].

Laryngeal tuberculosis responds well to antitubercular treatment. A six-month course of ATT is sufficient and provides remarkable results [15]. If laryngeal tuberculosis is not treated early, it may lead to glottic stenosis, subglottic stenosis, laryngeal muscles involvement, and vocal cord palsy due to invasion of recurrent laryngeal nerve or cricoarytenoid joint. Tubercular lesions of the larynx may cause fibrosis of the lamina propria of the vocal cords, leading to irreversible changes in voice quality [16]. Empirical steroid therapy in laryngeal tuberculosis may lead to flaring of the underlying tuberculosis, and it should be avoided.

\section{CONCLUSIONS}

Isolated or primary laryngeal tuberculosis among children is rare in routine clinical practice. It should be considered as a differential diagnosis in the case of any ulcerative or any inflammatory swelling of the vocal fold. Abnormal chest X-ray showing features of tuberculosis along with laryngeal lesion suggests the diagnosis, whereas normal chest X-ray cannot exclude the diagnosis. Therefore, paediatricians or otolaryngologists should keep in mind the existence of primary laryngeal tuberculosis to avoid delayed diagnosis and treatment.

\section{DISCLOSURE}

The authors declare no conflict of interest.

\section{REFERENCES}

1. World Health Organization. Global tuberculosis control. WHO Report 2010. Geneva 2010.

2. Uslu C, Oysu C, Uklumen B. Tuberculosis of the epiglottis: A case report. Eur Arch Otorhinolaryngol 2008; 265: 599-601.

3. World Health Organisation. Global tuberculosis report 2013. Geneva 2013. Available from: http://www.who.int/tb/publications/ global_report/en/ (Accessed: 21.05.2014).

4. World Health Organization. Guidance for national tuberculosis programmes on the management of tuberculosis in children. Geneva 2014. Available from: http://www.who.int/tb/publications/ childtb_guidelines/en/ (Accessed: 21.05.2014).

5. Singh K, Kaur G, Parmar TL. Pseudo tumoral laryngeal tuberculosis. India Paediatr 2003; 40: 49-52.

6. Unal M, Vayisoglu Y, Guner N, et al. Tuberculosis of the aryepiglottic fold and sinus pyriformis. Mount Sinai J Med 2006; 73: 806-809.

7. Shin JE, Nam SY, Yoo SJ, et al. Changing trends in clinical manifestations of laryngeal tuberculosis. Laryngoscope 2000; 110: 1950-1953.

8. Gassab E, Kedous S, Berkaoui A, et al. Extra-nodal head and neck tuberculosis. J Tun Orl 2010; 24: 26-30.

9. Lam PKY, Wei W. Medialisation thyroplasty for unilateral vocal fold paralysis associated with chronic pulmonary tuberculosis. Hong Kong Med J 2007; 13: 327-329.

10. Harney M, Hone S, Timon C, et al. Laryngeal tuberculosis: An important diagnosis. J Laryngol Otol 2000; 114; 878-880.

11. Nishiike S, Irigune M, Kubo T. Laryngeal tuberculosis: A report of 15 cases. Ann Otol Rhinol Laryngol 2002; 111: 916-918.

12. Prstacic R, Jurlina M, Zizic-Mitrecic M, Janjanin S. Primary nasopharyngeal tuberculosis mimicking exacerbation of chronic rhinosinusitis. J Laryngol Otol 2011; 125: 747-749. 
13. Eguchi J, Ishihara K, Watanabe A, et al. PCR method is essential for detecting Mycobacterium tuberculosis in oral cavity samples. Oral Microbiol Immunol 2003; 18: 156-159.

14. Menon K, Bem C, Gouldesbrough D, Strachan DR. A clinical review of 128 cases of head and neck tuberculosis presenting over a 10-year period in Bradford, UK. J Laryngol Otol 2007; 121: 362-368.

15. Lim JY, Kim KM, Choi EC, et al. Current clinical propensity of laryngeal tuberculosis: review of 60 cases. Eur Arch Otorhinolaryngol 2006; 21: 122-126.

16. Ozudogru E, Cakli H, Altuntas EE, et al. Effects of laryngeal tuberculosis on vocal fold functions: Case report. Acta Otorhinolaryngol Ital 2005; 25: 374-377. 